\title{
Optical supercomputing: introduction to special issue
}

\author{
Shlomi Dolev • Tobias Haist • Mihai Oltean
}

Published online: 16 May 2012

(C) Springer Science+Business Media, LLC 2012

Optical computers use photons rather than electrons to represent and modify information. Computer architectures that are based on optics offer several interesting features:

- Current architectures use energy to move electrons while photons move by nature. Thus, an optical information processing devices, consisting of passive components, may not use energy. Transport of information is accomplished with high speed and exceptional good efficiency and without crosstalk.

- Transmission of information over long distances is done by using light transmitted in fiber optics. Thus, there exists an inherent bottleneck of light to electronics conversion when information processing is done by electrons.

- High spatial parallelism.

- High density of information represented in 3D (holography) using free space or crystals.

- Some specific information tasks are already naturally solved by optical devices.

Still, optical computer technology is by far less studied, developed and actually used, with relation to electronic computing technology. Research is needed in order to make optics a viable replacement for electronics in the future.

\footnotetext{
S. Dolev

Ben-Gurion University of the Negev, P.O.B. 653, Be'er Sheva 84105, Israel

e-mail:dolev@cs.bgu.ac.il

T. Haist

Institute fuer Technische Optik, University of Stuttgart, Pfaffenwaldring 9, 70569 Stuttgart, Germany e-mail: haist@ito.uni-stuttgart.de
}

M. Oltean ( $₫)$

Babeş-Bolyai University of Cluj-Napoca, Kogalniceanu 1, Romania

e-mail: moltean@cs.ubbcluj.ro 
The International Workshop on Optical SuperComputing is a new annual forum for research presentations on all facets of optical computing for solving hard computation tasks. The first edition took place in Vienna, Austria and the papers were published by Springer LNCS [2].

The second edition of OSC took place in 2009 in Bertinoro, Italy [1]. Nineteen regular and invited papers have been presented during the workshop. The topics of the discussed papers included both theoretical and practical approaches, also with contributions to the use of optics in the scope of quantum computing and artificial intelligence [3]. The proceedings was published by Springer LNCS [3].

A post-conference special issue has been accepted at the Journal of SuperComputing. The following seven papers have been selected for inclusion in this issue:

- D. Fey, M. Schneider, J. Jahns and H. Knuppertz, Optical Multiplexing Techniques for Photonic Clos Networks in High Performance Computing Architectures, where the authors investigate how a photonic on-board Clos network can be realized using Coarse Wavelength-Division-Multiplexing (CWDM) techniques with components based on fiber technology. A new photonic Clos network on chip, which reduced the number of switches, is also presented.

- D.E. Tamir, N.T. Shaked, W.J. Geerts and S. Dolev, Parallel Decomposition of Combinatorial Optimization Problems using Electro-Optical Vector by Matrix Multiplication Architecture, surveys structures for representing Hamiltonian cycles, the use of these structures in heuristic optimization techniques, and efficient mapping of these structures along with respective operators to a newly proposed electro-optical vector by matrix multiplication architecture.

- T. Haist and W. Osten, White-Light Interferometric Method for Secure Key Distribution, proposes a protocol for secure key distribution using classical optics. In this way single photons (and the practical problems associated with them) are avoided. The method ensures that shared random keys are securely created, thus enabling secure data transmission.

- S. Goliaei and S. Jalili, An Optical Solution to the 3-SAT Problem using Wavelength Based Selectors, which describes a device for solving an NP-complete problems by dividing and filtering wavelengths. The method considers each group of wavelengths as a possible value-assignment for the variables. Optical filters to drop wavelengths not satisfying the formula from the light ray are employed. At the end, remaining wavelengths indicate all possible assignments for satisfying the formula.

- A. Zlotnik, M. Paturzo, P. Ferraro and Z. Zalevsky, Optical Spatial Image Processor based on Aliasing of Pseudo-Periodic Sampling, presents a new configuration for a real-time spatial image processor that is based upon a spatially incoherent imaging setup in which a grating is attached to the object plane.

- H.J. Caulfield, A. Zavalin, and L. Qian, Zero-Energy Optical Logic: Can it be Practical?, which shows how a lossless logic systems could actually be built. The authors propose that the only way in which such system can be built is optics.

- X. Li, W. Hu, H. Zhang, Y. Nie, J. Yang and J. Yang, Operation Speed Limited by the Electric Properties of the Photorefractive Spatial Light Modulator, which analyzes the current pulses in order to infer characteristics and to find out the relationship between the properties of the photo-induced current pulses and the structure parameters of the SLM. 
These articles represent different approaches for optical supercomputing. We look forward to the continuation and developing of this new exciting, interesting and very promising field of research.

Shlomi Dolev acknowledges support from Rita Altura Trust Chair in Computer Sciences. Mihai Oltean was supported by Grant IDEI 2412/2008 from CNCSIS, Romania.

\section{References}

1. Website for optical SuperComputing (2009). http://www.cs.bgu.ac.il/ dolev/OSC09

2. Dolev S, Haist T, Oltean M (eds) (2008) Optical Super Computing, First International Workshop OSC 2008, Proceedings, Vienna, Austria, August 26, 2008. Lecture Notes in Computer Science, vol 5172. Springer, Berlin

3. Dolev S, Oltean M (eds) (2009) Optical Super Computing, The Second International Workshop, OSC 2009, Proceedings, Bertinoro, Italy, November 18-20, 2009. Lecture Notes in Computer Science, vol 5882. Springer, Berlin 